\title{
Time Domain Analysis of a Common-Mode Suppression Filter Subjected to a Multi-Objective Optimization
}

\author{
Celina Gazda, Hendrik Rogier, and Dries Vande Ginste \\ Electromagnetics Group \\ Department of Information Technology \\ Ghent University \\ Sint-Pietersnieuwstraat 41 \\ 9000 B-Gent, Belgium \\ Email: celina.gazda@intec.UGent.be
}

\author{
Ivo Couckuyt and Tom Dhaene \\ Surrogate Modeling Lab \\ Department of Information Technology \\ Ghent University-IBBT \\ Gaston Crommenlaan 8 \\ 9050 B-Gent, Belgium
}

\begin{abstract}
An optimal common-mode suppression filter for a bend discontinuity in a pair of differential lines is characterized simultaneously by a large reduction of conversion noise, a small differential mode reflection coefficient and low overall loss, taking into account technology constraints. To find the geometrical parameters of such optimal design, a novel constrained multiobjective optimization method, relying on intermediate surrogate models of several costs, is proposed. A 3D Pareto-front is generated and further, constrained to impose hardware limitations. The front shows trade-offs between the different cost functions, as such allowing to choose an optimal solution from a large set of available layouts. As is often the case, the filter design occurs in the frequency domain. Additionally, in this contribution the method is also validated by means of a time domain analysis, confirming its accuracy and efficiency.
\end{abstract}

\section{INTRODUCTION}

Modern high-speed digital circuits often use coupled microstrip lines to transmit differential signals across boards, as this way of signaling is characterized by high electromagnetic (EM) immunity against conducted and radiated noise, low EM interference and crosstalk. To avoid severe signal integrity degradation [1] and high noise levels at the receiving end, threatening the safety of the receiver, a low level of differential-to-common mode conversion is required. Unfortunately, mode conversion noise is induced easily at every discontinuity, and in particular at a bend [2]

One of many possible filter designs that can be applied in the area of the bend to minimize mode conversion is the new structure described in [2], [3], where a very good suppression of common-mode noise was achieved, although less attention was devoted to minimize reflection and losses at the same time. Therefore, in this paper we apply a new multiobjective optimization [4] method to account for these conflicting requirements. To speed up the optimization process, which usually relies on a large number of time-consuming full-wave simulations, we propose a novel approach by constructing and exploiting multiple surrogate models in a three-step strategy. First, surrogate models are simultaneously constructed for six cost functions. Thereto, only one limited set of full-wave simulations is needed. Second, a 3D Pareto-front is created by means of multi-objective optimization relying on the generated surrogates. Third, the Pareto-front is reduced to an acceptable region satisfying hardware constraints, leveraging again the surrogate models. Once constructed, this constrained Paretofront allows a designer to quickly decide which design from the set of available layouts best fits the actual system requirements within the set of constraints imposed by the hardware and the manufacturing process.

It is a common practice for engineers to design filters in the frequency domain, e.g. by optimization of the pertinent frequency-dependent scattering parameters. Therefore, in [5], we based our optimization method of the common-mode suppression filter on the frequency-dependent modal scattering parameters. However, for the particular case of signaling across a pair of differential lines, time domain behavior of the interconnect is equally important. In this contribution we show that our proposed optimization technique also leads to excellent, reliable time domain results. This validation was performed by analyzing the common mode noise at the receiving end, differential mode noise reflected towards the transmitter and time domain transmissometry (TDT) eye diagrams.

In the next section we describe the filter, which is the subject of our optimization, together with its conflicting requirements, whereas Section III outlines the surrogate modeling based multi-objective optimization strategy. In Section IV, the procedure is further detailed and applied to the common mode suppression filter. Time domain results of three carefully chosen samples are given in Section $\mathrm{V}$, to prove the accuracy and efficiency of the proposed method. The last section summarizes our work.

\section{COMMON-MODE SUPPRESSION FILTER}

In a pair of coupled lines differential-to-common mode conversion does not occur as long as the structure remains 
symmetrical. However, in real printed circuit boards applications, it is impossible to avoid discontinuities such as bends. In a classic $90^{\circ}$ bend, consisting of a pair of coupled microstrip lines with a cross-section as in Fig. 1(a), designed on a Rogers RO4350B substrate $\left(\epsilon_{r}=3.66, \tan \delta=0.003\right.$, $h=1.524 \mathrm{~mm}, t=35 \mu \mathrm{m}$, and $\sigma=4.1 \cdot 10^{7} \mathrm{~S} / \mathrm{m}$, which is the value for the gold used to plate the copper microstrip lines on the board), the outer line is much longer than the inner one (Fig. 1(b)), causing time delay between the propagating differential signals. This introduced time skew is the main reason for differential-to-common mode conversion and unwanted noise. The structure is designed to have a characteristic impedance of $50 \Omega$ for the odd mode. The characteristic impedance for the even mode is still too small $(87.47 \Omega)$ to provide a difference between the impedances that is large enough to sufficiently prevent the differential mode from converting to the common mode.

To alleviate this problem, we proposed to locally modify the coupled microstrip lines to tightly coupled ones in the area of the bend using tapering sections (Fig. 1(c)). The relevant geometrical parameters of this design are: cross-section of the classic coupled microstrip lines (line width $w_{1}$ and spacing $\left.s_{1}\right)$, cross-section of the tightly coupled microstrip lines $\left(w_{2}\right.$ and $s_{2}$ ), lengths $l_{1}$ and $l_{2}$ of the classic coupled microstrip lines and the tightly coupled section, respectively, and the length $l_{t}$ of the tapers. The total length of the structure is fixed to $L=l_{1}+l_{t}+l_{2}$. This solution decreases the length difference between the two traces, significantly reducing the time delay between propagating signals, which leads to the reduction of the differential-to-common mode conversion. Moreover, the higher coupling factor between the lines and higher even mode impedance in the area of the bend, while the odd mode impedance remains matched to $50 \Omega$, makes the structure act as a natural common-mode suppression filter. The applicability of the proposed structure to common mode filtering is verified in [2] and [3], where the main focus was on the mode conversion and differential mode transmission and reflection. However, other characteristics such as overall losses, manufacturing tolerance, compact size, etc. are also important. Since some of these objectives are conflicting, a perfect geometry providing overall optimal performance does not exist. A longer section of tightly coupled lines (larger $l_{2}$ ) prevents direct coupling between the tapers but it increases the overall losses. More tightly coupled lines in the area of the bend (smaller $w_{2}$ and $s_{2}$ ) help to reduce mode conversion but again increase total loss and reflection. Moreover, they make the structure more sensitive to production tolerances. Since the ideal solution cannot be found, we need to select the most important objectives and perform a multi-objective optimization to find the set of optimal structures.

\section{Multi-Objective Optimization}

Multi-objective optimization methods that rely on evolutionary algorithms need a lot of samples to operate, which may be prohibitively expensive in the case of complex CPU-time intensive simulation codes. Relying on surrogate modeling is a

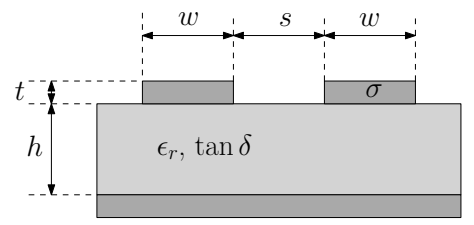

(a) Cross-section

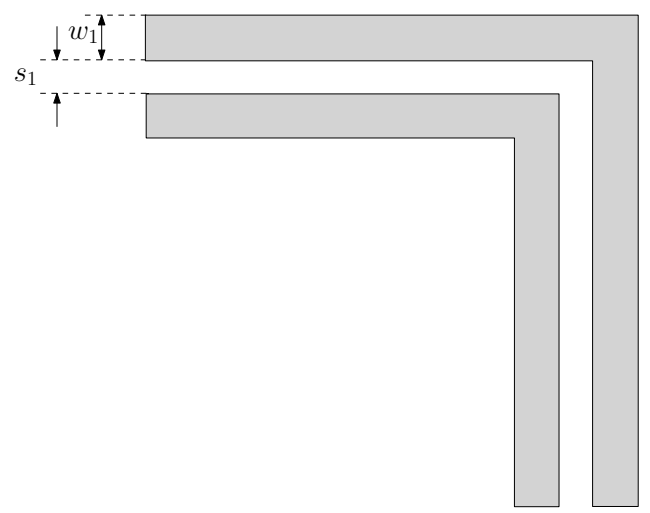

(b) Top view of classic $90^{\circ}$ bend

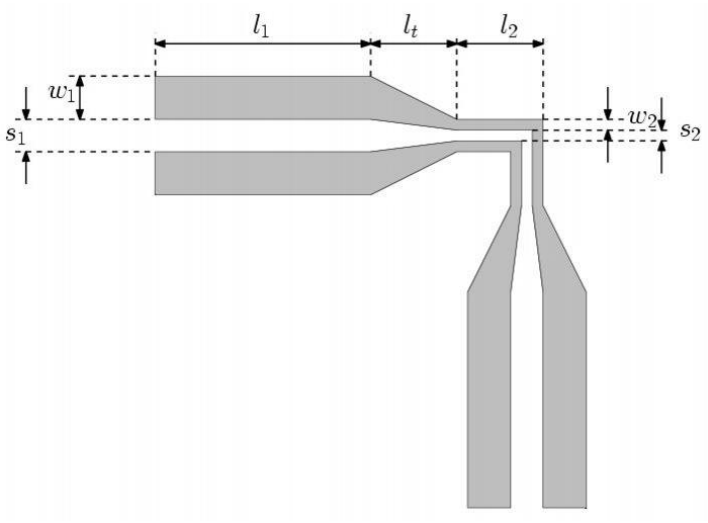

(c) Top view of common-mode suppression filter

Fig. 1. Classic bend and common-mode suppression filter with their geometrical parameters.

common technique to reduce the total simulation time, as this reduces the number of required full-wave models. The idea of using surrogates for the objectives in order to expedite the optimization process is used in global surrogate modeling [6] and Multi-Objective Surrogate-Based Optimization (MOSBO) [7], [8]. A surrogate is a cheap approximation of a complex simulation model that can be used to replace that simulation model, allowing to quickly obtain any number of additional function evaluations without resorting to more expensive numerical models. In the optimization process, the surrogate models are used to create a globally accurate approximation of each cost function so that the multi-objective optimization algorithm is applied using the surrogates instead of the expensive simulation code. The initial extra cost in constructing the surrogate models is later on more than compensated for, as each full-wave simulation can be reused in the simultaneous construction of all cost functions required to generate a very 
dense Pareto-front of optimal solutions and to subsequently evaluate additional constraints imposed by hardware.

\section{Constrained Pareto Optimization of the Filter}

To find the optimal geometrical parameters of the commonmode suppression filter, e.g. in the frequency band from DC to $6 \mathrm{GHz}$, using $L^{p}$-norms [9] we define six cost functions as follows:

$$
\begin{aligned}
\text { cost }_{1}= & {\left[\int_{0 \mathrm{GHz}}^{6 \mathrm{GHz}}\left|s_{d d 11}(f)\right|^{2} d f\right]^{1 / 2} } \\
\text { cost }_{2}= & {\left[\int_{0 \mathrm{GHz}}^{6 \mathrm{GHz}}\left(\left|s_{c d 11}(f)\right|^{2}+\left|s_{c d 21}(f)\right|^{2}\right) d f\right]^{1 / 2} } \\
\text { cost }_{3}= & {\left[\int_{0 \mathrm{GHz}}^{6 \mathrm{GHz}}\left(P_{\text {loss }}(f)\right) d f\right]^{1 / 2} } \\
= & {\left[\int _ { 0 \mathrm { GHz } } ^ { 6 \mathrm { GHz } } \left(1-\left|s_{d d 11}(f)\right|^{2}-\left|s_{d d 21}(f)\right|^{2}\right.\right.} \\
\text { cost }_{4}= & \max _{f}\left|s_{d d 11}(f)\right| \\
\text { cost }_{5}= & \max _{f} \sqrt{\left|s_{c d 11}(f)\right|^{2}+\left|s_{c d 21}(f)\right|^{2}} \\
\text { cost }_{6}= & \max _{f} \sqrt{P_{\text {loss }}(f)}
\end{aligned}
$$

where the quantities $s_{d d 11}, s_{d d 21}, s_{c d 11}$ and $s_{c d 21}$ indicate the pertinent frequency-dependent modal scattering parameters. The costs (1)-(3), obtained using $L^{2}$-norms $(p=2)$, correspond to the mean-squared frequency average of the differential reflection coefficient, the total differential-to-common mode conversion and the total losses, respectively. The costs (4)-(6) calculated using $L^{\infty}$-norms $(p=\infty)$, provide the maximum values of these parameters.

In a first step, relying on a single set of 100 full-wave simulations, six surrogate models of the mentioned cost functions are constructed using least-squares support vector regression (SVR) [10] with a lola-voronoi sampling algorithm. The samples are selected by the lola-voronoi sample selector [11], evaluated and SVR models are updated until their crossvalidation decreases below $10^{-4}$, which provides sufficient accuracy for the surrogate models to be used as a starting point in a multi-objective optimization (Fig. 2). The planar3D full-wave simulator ADS-Momentum was used to obtain full-wave results. The geometrical parameters $w_{2}$ (with $s_{2}$ chosen to provide a $50 \Omega$ odd-mode impedance) and $l_{2}$ were varied over $[0.1,1] \mathrm{mm}$ and $[2,8] \mathrm{mm}$, respectively. Each cost function is approximated independently using a least-squared SVR surrogate model [12]. A computer with a $3 \mathrm{GHz}$ Quad CPU and with 8 GB RAM needed $5 \mathrm{~h} 2 \mathrm{~min}$ in total to build all models. Fig. 3 presents the surrogate models for (1)-(3) based on the $L^{2}$-norm. A higher differential-mode reflection but also a better common-mode suppresion are found for

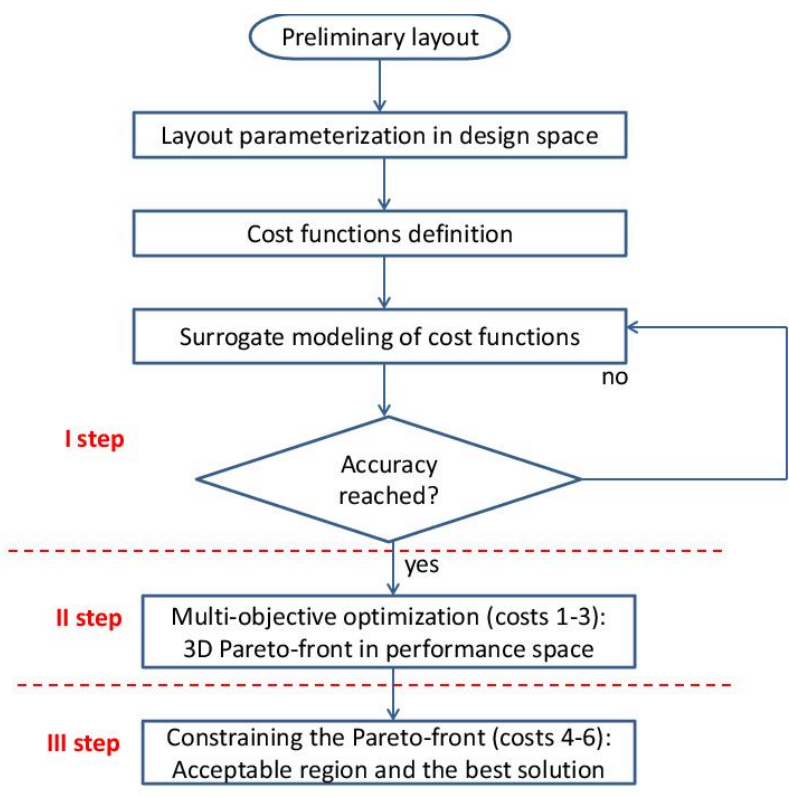

Fig. 2. Flow chart of the three-step optimization process.

smaller $w_{2}$ (a more tightly coupled cross-section in the area of the bend), as proven in Figs. 3(a) and 3(b), respectively. Fig. 3(c) confirms that overall losses increase for increasing $l_{2}$ and decrease with increasing $w_{2}$.

Second, the multi-objective optimization is performed (Fig. 2) using the SPEA2 algorithm [13] with a population size of 600 for 200 generations, to simultaneously minimize all three cost functions for the $L^{2}$-norms (10 min $2 \mathrm{~s}$ on the same machine). If the full-wave simulations were not replaced by the intermediate surrogate models, the complete optimization time would be about $25 \mathrm{~h} 12 \mathrm{~min}$ (600 runs, each one taking 2 min 31 s). Fig. 4(a) shows the 3D Pareto-front [14] in the performance space, restricted by the ranges over which $w_{2}$ and $l_{2}$ may vary given the technology constraints. Fig. 4(b) represents the corresponding geometrical parameters of the Pareto-front samples in the design space (for clarity, in Fig. 4 only 250 samples out of 600 are shown). Corner $\{1\}$ of the Pareto-front indicates samples with both $w_{2}$ and $l_{2}$ small (very short and very tightly coupled lines in the area of the bend) that provide very high mode conversion suppression, but also large reflection and high power losses. In corner $\{2\}$ of the front, conversion loss remains very small while the differential mode reflection decreases and the total losses increase as $l_{2}$ increases. Differential mode reflection coefficient and total losses are largely reduced while the conversion loss increases in corner $\{3\}$, as the lines in the area of the bend are short and weakly coupled.

Third, to reject designs that might be harmful for the transmitter and/or receiver, additional constraints are imposed to the Pareto-front (Fig. 2), using the surrogate models for the 


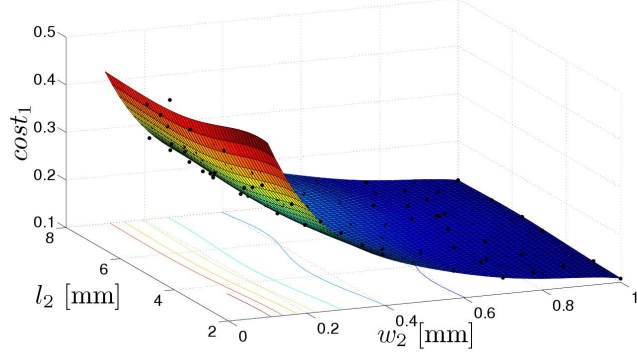

(a) Surrogate model for $\operatorname{cost}_{1}\left(L^{2}\right.$-norm).

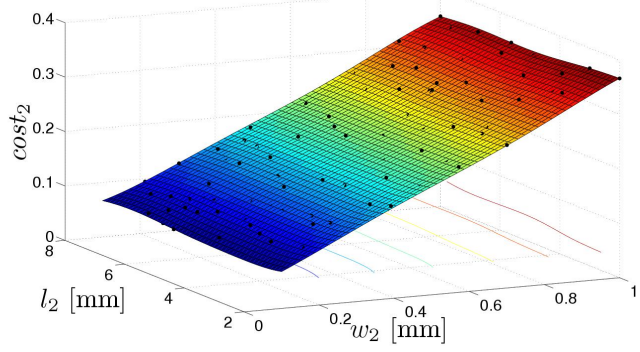

(b) Surrogate model for $\operatorname{cost}_{2}\left(L^{2}\right.$-norm).

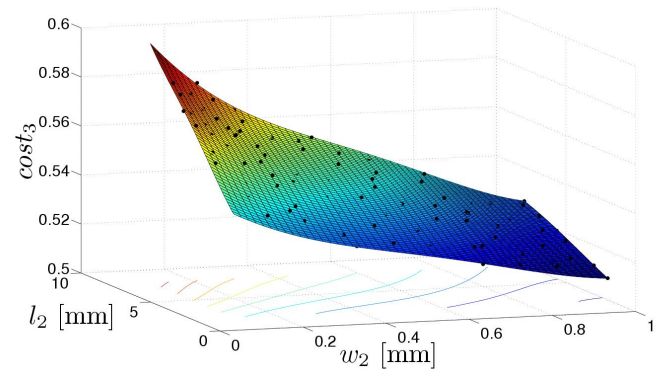

(c) Surrogate model for $\operatorname{cost}_{3}\left(L^{2}\right.$-norm).

Fig. 3. Surrogate models for the three cost functions of the chosen objectives (black dots: 100 full-wave simulation samples; surface plot: surrogate model).

$L^{\infty}$-norms:

$$
\begin{aligned}
& \text { constraint }_{1}: \operatorname{cost}_{4}<-15 \mathrm{~dB} \\
& \text { constraint }_{2}: \operatorname{cost}_{5}<-15 \mathrm{~dB} \\
& \text { constraint }_{3}: \operatorname{cost}_{6}<8 \%
\end{aligned}
$$

The green circles (o) on the Pareto-front represent designs satisfying all three constraints. Blue squares $(\square)$, pink leftpointing triangles $(\triangleleft)$ and dark violet triangles $(\triangle)$ indicate layouts that violate the first, second and third constraint, respectively. Orange diamonds $(\diamond)$ depict layouts violating both first and third constraint. The sample labeled $\mathrm{A}$ is the sample with geometry $w_{2}=0.45 \mathrm{~mm}, s_{2}=0.18 \mathrm{~mm}$ and $l_{2}=2.16 \mathrm{~mm}$ that provides the best solution in terms of minimal averaged insertion loss $<\mathrm{IL}\rangle$, obtained when all three costs are equally important, as follows: $<\mathrm{IL}>=$ $\operatorname{cost}_{1}^{2}+\operatorname{cost}_{2}^{2}+\operatorname{cost}_{3}^{2}$, using the $L^{2}$-norm for the costs i.e. (1), (2) and (3). This might be the designer's final choice.

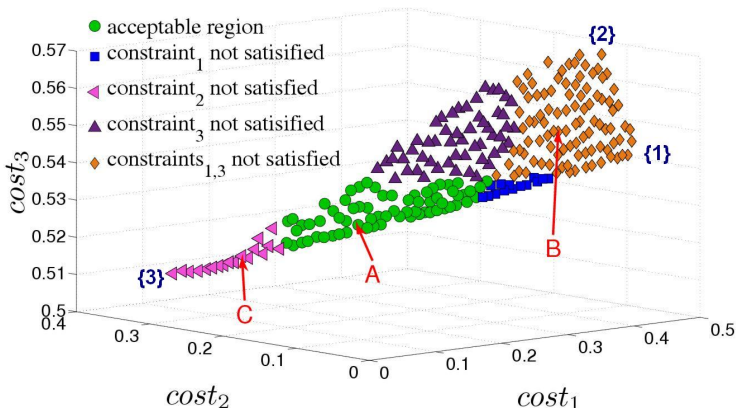

(a) 3D Pareto-front in the performance space for the three cost functions.

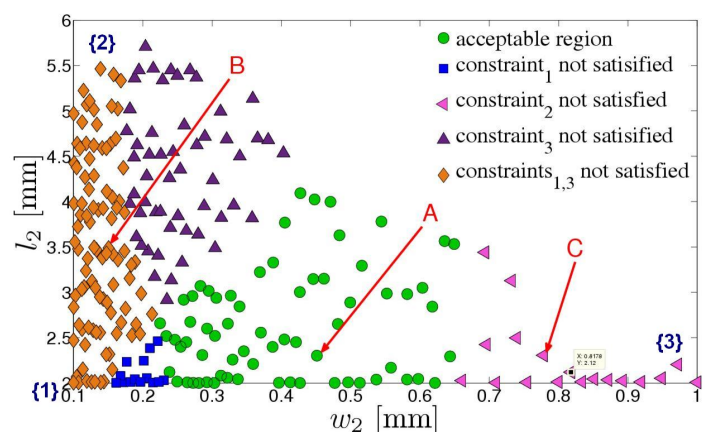

(b) Pareto samples in the design space.

Fig. 4. 3D Pareto-front and its corresponding geometrical parameters (for readability, only 250 out of 600 samples are shown).

\section{TIME DOMAIN RESUlTS}

To validate the results obtained by the above described strategy, three samples from three different groups of the Pareto-front (Fig. 4(a)) were selected and analyzed in the time domain. Sample A is the suggested optimal solution with the geometry mentioned above, sample B is one of the orange diamonds $\left(w_{2}=0.15 \mathrm{~mm}, s_{2}=0.13 \mathrm{~mm}\right.$, $\left.l_{2}=3.49 \mathrm{~mm}\right)$ and sample $\mathrm{C}$ is one of the pink left pointing triangles $\left(w_{2}=0.82 \mathrm{~mm}, s_{2}=1.43 \mathrm{~mm}, l_{2}=2.12 \mathrm{~mm}\right)$. First, an analysis of noise introduced by mode conversion and differential mode reflection was performed. A ramped step signal is applied to the input port of the outer line, going from $0 \mathrm{~V}$ up to $1 \mathrm{~V}$ with a rise time of $t_{r}=30 \mathrm{ps}$, while at the input port of the inner line a ramped step signal is going from $0 \mathrm{~V}$ down to $-1 \mathrm{~V}$ with a fall time of $t_{f}=30 \mathrm{ps}$. The output ports were matched to $50 \Omega$. Fig. 5 shows that sample B performs the best, inducing only $20 \mathrm{mV}$ of the common mode noise at the receiving end. However, Fig. 6 shows that the same sample exhibits the highest reflected differential mode noise $(135 \mathrm{mV})$, making it an unacceptable choice. For sample $\mathrm{C}$ the opposite behavior is noticed, yielding $80 \mathrm{mV}$ of common mode noise at the receiver (Fig. 5), while causing only $28 \mathrm{mV}$ of reflected differential mode noise. Only sample A, with $50 \mathrm{mV}$ of common mode noise and $53 \mathrm{mV}$ of reflected differential mode noise (Figs. 5 and 6, respectively) remains within acceptable limits.

Next, the input signal was replaced by a pseudo-random 


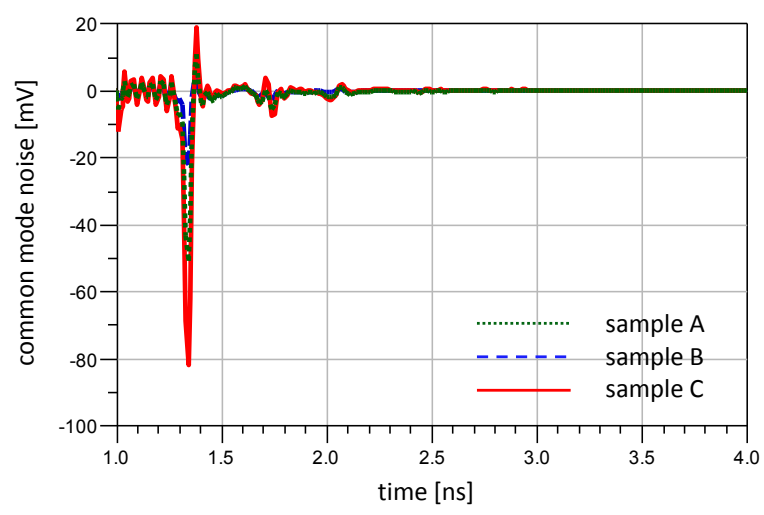

Fig. 5. Common mode noise at the receiver for the three bends.

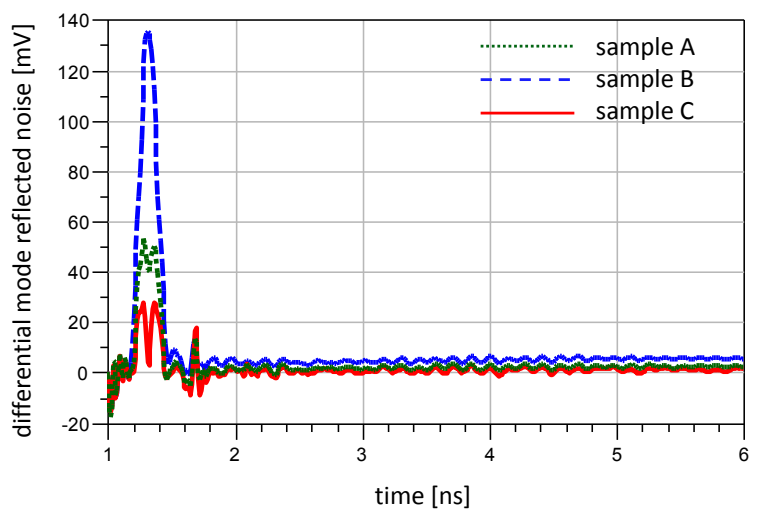

Fig. 6. Differential mode reflected noise at the transmitter for the three bends.

bit sequence with a bit rate of $10 \mathrm{Gbps}$ and a rise/fall time $t_{r / f}=30 \mathrm{ps}$ and TDT eye diagrams (Fig. 7) were created that confirm the previous conclusions. Since the eye diagrams illustrate the quality of the signal at the receiver, sample B provides the eye with the largest opening (Table I) and could be considered as the best result. As stated above, however, this sample exhibits a large reflected differential mode noise. The eye diagram of the sample $\mathrm{C}$ creates the largest amount of the common mode noise and as such it has the smallest eye in terms of its width and height. Sample A, being the optimal solution of the trade-off between common mode noise and differential mode reflected noise, provides a very good and clear eye diagram and it can be considered as an optimal

TABLE I

EYE DIAGRAM PARAMETERS

\begin{tabular}{|c||c|c|c|}
\hline parameter & sample A & sample B & sample C \\
\hline \hline height & $0.795 \mathrm{~V}$ & $0.815 \mathrm{~V}$ & $0.757 \mathrm{~V}$ \\
\hline width & $95.8 \mathrm{ps}$ & $98.8 \mathrm{ps}$ & $95 \mathrm{ps}$ \\
\hline
\end{tabular}

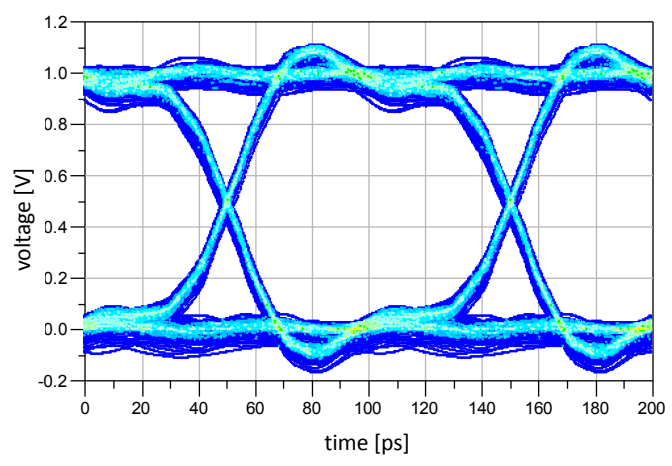

(a) Sample A

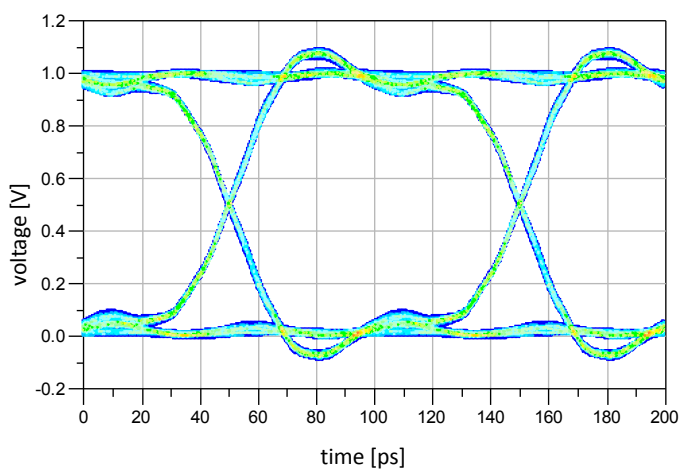

(b) Sample B

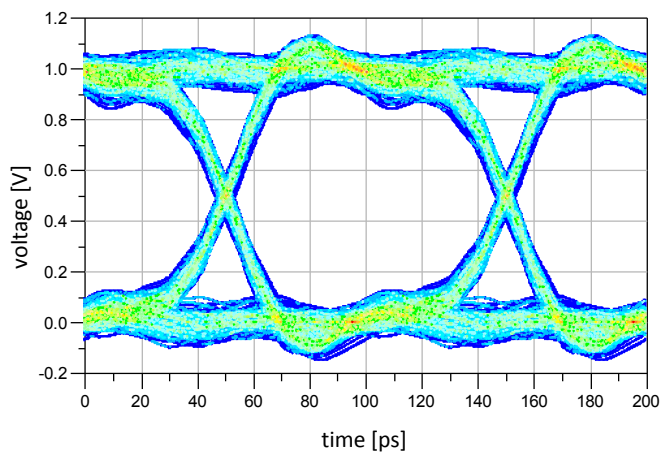

(c) Sample C

Fig. 7. Eye diagrams for the three chosen samples.

solution. The above observations for samples A, B, and C, derived from Figs. 5-7, comply with the location of these samples on the constrained Pareto-front.

\section{CONCLUSION}

A constrained multi-objective optimization strategy, using intermediate surrogate models of the cost functions, that determines the optimal parameter set of layouts for a commonmode suppression filter has been presented. The 3D Pareto- 
front, representing the set of feasible optimal designs, is restricted to account for hardware constraints. The presented methodology aids the EMC-aware designer in efficiently visualizing the design space and selecting an optimal solution given conflicting costs while respecting the hardware limits. Time domain analysis performed on three different samples confirm the validity and efficiency of this method, showing that the sample taken from the acceptable region of the Paretofront performs the best in terms of simultaneously reducing the common-mode noise and the reflected differential-mode noise on the one hand and providing a maximal throughput of the differential signal on the other.

\section{REFERENCES}

[1] J. Fan, X. Ye, J. Kim, B. Archambeault, and A. Orlandi, "Signal integrity design for high-speed digital circuits: Progress and directions," IEEE Trans. on EMC, vol. 52, no. 2, pp. 392 -400, May 2010.

[2] C. Gazda, D. Vande Ginste, H. Rogier, R.-B. Wu, and D. De Zutter, "A wideband common-mode suppression filter for bend discontinuities in differential signaling using tightly coupled microstrips," IEEE Trans. Adv. Pack., vol. 33, no. 4, pp. 969-978, Nov. 2010.

[3] C. Gazda, D. Vande Ginste, H. Rogier, D. De Zutter, and R.-B. Wu, "Time domain analysis of a wideband common-mode suppression filter for bent interconnects," in 15th IEEE Workshop on Signal Propagation on Interconnects (SPI), Naples, Italy, 8-11 May 2011, pp. 7 -10.

[4] Y. Li, V. Jandhyala, and H. Braunisch, "Multi-objective optimization of microprocessor package vertical interconnects," in IEEE 61st Electronic Comp. and Tech. Conf. (ECTC), Jun 2011, pp. 495-500.

[5] C. Gazda, I. Cockuyt, H. Rogier, D. Vande Ginste, and T. Dhaene, "Constrained multi-objecitve optimization of a common-mode suppression filter," Accepted for publication in IEEE Trans. on EMC, 2012.

[6] D. Gorissen, K. Crombecq, I. Couckuyt, P. Demeester, and T. Dhaene, "A surrogate modeling and adaptive sampling toolbox for computer based design," Journal of Machine Learning Research, vol. 11, pp. 2051-2055, Jun. 2010.

[7] G. Wang and S. Shan, "Review of metamodeling techniques in support of engineering design optimization," Journal of Mechanical Design, vol. 129, no. 4, pp. 370-380, Apr. 2007.

[8] I. Couckuyt, F. Declercq, T. Dhaene, H. Rogier, and L. Knockaert, "Surrogate-based infill optimization applied to electromagnetic problems," International Journal of RF and Microwave Computer-Aided Engineering, vol. 11, no. 5, pp. 492-501, 2010.

[9] D. Zwillinger and C. R. Company, CRC standard mathematical tables and formulae, 31st ed. CRC Press, 2003, ch. 1, pp. 72-73.

[10] J. Suykens, T. Van Gestel, J. De Brabanter, B. De Moor, and J. Vandewalle, Least Squares Supported Vector Machines. World Scientific Publishing Co, 2002.

[11] K. Crombecq, L. De Tommasi, D. Gorissen, and T. Dhaene, "A novel sequential design strategy for global surrogate modeling," in Proc. of the 2009 Winter Simulation Conference, Austin, USA, 13-16 dec. 2009, pp. $731-742$.

[12] K. Crombecq, D. Gorissen, D. Deschrijver, and T. Dhaene, "A novel hybrid sequential design strategy for global surroagte modeling of computer experiments," Journal on Scientific Computing, vol. 33, no. 4, pp. 1948-1974, Jul-Aug 2011.

[13] E. Zitzler, M. Laumanns, and L. Thiele, "SPEA2: Improving the strength pareto evolutionary algorithm for multiobjective optimization," in Evolut. Methods for Dsn Opt. and Ctrl with Appl. to Indstr. Prob. Int. Ctr for Num. Methods. in Eng., 2001, pp. 95-100.

[14] Y. Rahmat-Samii and E. Michielssen, Eds., Electromagnetic Optimization by Genetic Algorithms, 1st ed. John Wiley \& Sons, Inc., 1999, ch. 2 , pp. $48-49$. 